\title{
Human herpesvirus 6 infection as a trigger of multiple sclerosis: an update of recent literature
}

\author{
K. I. Voumvourakis ${ }^{1}$, P.C. Fragkou ${ }^{2}$, D. K. Kitsos ${ }^{1}$, K. Foska ${ }^{1}$, M. Chondrogianni ${ }^{1}$ and S. Tsiodras ${ }^{2}$
}

\begin{abstract}
Background: This is an update on the existing evidence regarding a relationship between infection with human herpesvirus 6 (HHV-6) and multiple sclerosis (MS) in order to contribute on the attempt to define the nature and strength of that relationship.

Results: Study quality was assessed using the criteria proposed by Moore and Wolfson and by the classification criteria used by the Canadian Task Force on the Periodic Health Examination. Studies were categorized both by experimental technique and by quality (high [A], intermediate $[\mathrm{B}]$, and low $[\mathrm{C}]$ ) as determined by the Moore and Wolfson criteria. Overall, 27 (90\%) of 30 studies, 18 (86\%) of which were classified as A quality, reached a statistically significant result. According to the Canadian Task Force classification, all studies were categorized as evidence of qualityll-1. Limitations of the available experimental techniques and perspectives for future research are discussed.
\end{abstract}

Conclusions: The current review continues to emphasize the need for further, objective, evidence-based examination of the relationship between HHV-6 infection and multiple sclerosis.

Keywords: Cerebrospinal fluid, CSF, Demyelination, Diagnosis, HHV-6, Human herpes virus 6,lgG antibodies, IgM antibodies, Multiple sclerosis, Pathogenesis,pcr, Polymerase Chain Reaction, Polymorphisms, Serology, Etiology

\section{Introduction}

The role of viral agents in the pathogenesis of Multiple Sclerosis (MS) has been a matter of intense scientific interest [1]. Although to date, no specific viral agent has been implicated as a definite causative factor of MS, infections have long been thought to be significant contributors to disease development or exacerbation [2]. Results of familial aggregation and migration studies indicate that exposure to environmental factors, both infectious and non-infectious, during childhood and adult life are strong determinants of MS risk [3]. Thus, viruses remain at the epicenter of the research for MS

\footnotetext{
*Correspondence: cvoumvou@outlook.com

1 2nd Department of Neurology, Attikon University Hospital, National

and Kapodistrian University of Athens, Athens, Greece

Full list of author information is available at the end of the article
}

pathogenesis for more than 40 years [2]. Figure 1 summarizes available data supporting a viral triggering effect on MS pathogenesis.

Among numerous viruses, Human herpesvirus 6 (HHV6), an ubiquitous pathogen that falls into latency and periodically reactivates, represents a plausible candidate as a causative or triggering factor owing to its neurotropic, lymphotropic and immunomodulatory characteristics [3]. Two variants of HHV-6 have been recognized: HHV-6A, with a more prominent preference for infecting neural cells, and HHV-6B [3, 4]. However, it is less often associated with disease than HHV-6B. HHV-6A has been found predominantly in MS lesions. Primary evidence backing a convincing pathogenic role for HHV-6 in MS was based on cerebrospinal fluid (CSF) detection of viral DNA by polymerase chain reaction (PCR). Observation of viral messenger RNA and protein expression in oligodendrocytes 


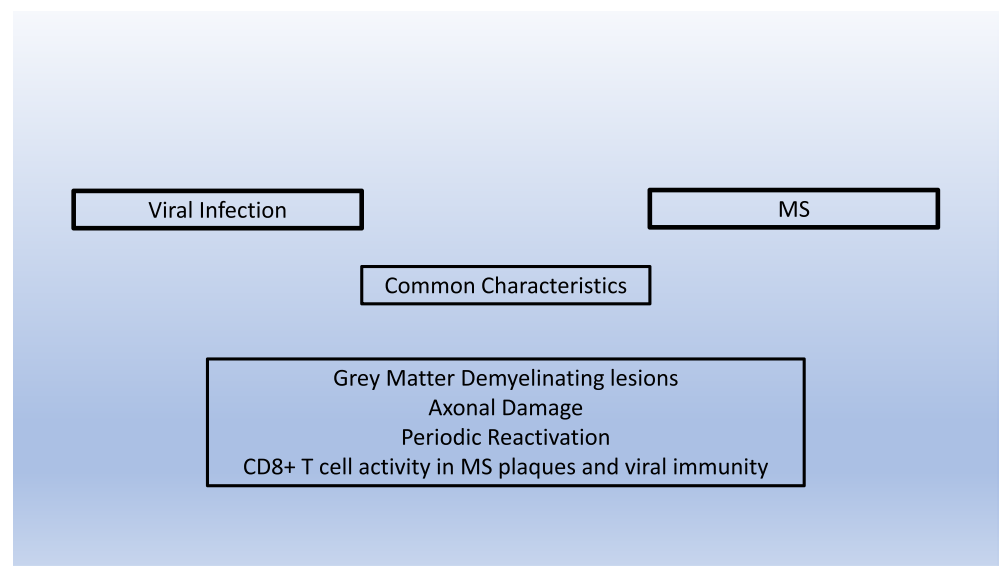

Fig. 1 Common characteristics of a viral infection and demyelination in the form of Multiple Sclerosis

were additional contribution to the hypothesis of HHV-6 as an operator of MS [4]. In this review, an update is presented regarding the evidence on the potential relationship between HHV-6 and MS pathogenesis [4] as well as a possible alteration in the immunological status of MS HHV-6 seropositive patients versus MS seronegative ones.

\section{Methods}

We conducted a review of clinical studies reporting the detection of HHV-6 genome or anti-HHV-6 antibody response in patients with MS. The authors independently performed the literature search, study selection and data extraction. Studies reporting serological, cerebrospinal fluid (CSF) and genome studies identifying HHV-6 activity in MS patients were included while HHV-6 preclinical data and animal studies were excluded from the literature search. No language restrictions were applied to the literature search. Pubmed MEDLINE database was accessed covering the period from $01 / 01 / 2010$ up to $30 / 06 / 2021$ using the following search terms: human herpes virus 6, HHV-6, demyelination, multiplesclerosis, pathogenesis, diagnosis, serology, cerebrospinal fluid, CSF, IgG antibodies, IgM antibodies, PCR, polymerase chain reaction and polymorphisms.Retrieved studies from the initial search were further screened for additional articles. The quality of the included trials was classifiedaccording to the criteria proposed by Moore and Wolfson as well as those proposed by the Canadian Task Force (CTF) on the Periodic Health Examination $[5,6]$. The studies with statistically significant correlation between MS and HHV-6 infection are presented in Table 1. The corresponding flow chart is presented on Fig. 2

\section{Results}

\section{Serological Studies}

In total, 9 studies reported the measurement of serum anti-HHV-6 IgG and/or IgM antibodies, in their methodology. Among these studies, 8 concluded on a statistically significant correlation between quantitative HHV-6 serology results and MS evolution as presented in Table 1. As an example, Ortega-Madueño et al. found that $69 \%$ of MS patients who were free of relapses and disease progressionhadlow anti-HHV-6A/B IgG titers after 2 years of different Disease Modifying Therapies (DMTs) compared to $40.7 \%$ with an increase of antibody titers $(p<0.001)$; the highest significance was notedin patients who received Natalizumab [7]. Natalizumab is an immune modulator that impairs the entry of $\mathrm{B}$ and plasma cells into the central nervous system (CNS) and, therefore, is associated with decreased total levels of IgG and IgM in CSF [8-10]. Additionally, OrtegaMadueño et al. found a peak ofanti-HHV-6A/B IgG and IgM titerstwo weeks and one-month, respectively, prior tothe occurrence of a clinical relapse [7]. Another study showed that the IgG antibodies against the latency associated HHV-6 protein (anti-HHV-6 U94/REP IgG) were higher in MS patients compared to healthy controls. In addition, a significant difference in anti-HHV-6 U94/REP immunoglobulin titers was depicted between the relapse and remission phases [11]. Similarly, a prospective cohort study demonstrated that anti-HHV-6 IgG titer was positively associated with the hazard of disease relapse with a dose-dependent trend [12]. However, the same authors found that this association was not mediated by the reactivation of HHV-6, as neither anti-HHV-6 IgM nor HHV-6 viral load were associated with relapse hazard, disability, or disability progressionin a subsequent study [13]. Another team that screened MS patients and controls for antibodies against numerousviral pathogens, found significantly higher seroprevalence of both antiHHV-6 IgG and IgM in MS patients compared to healthy controls [14]. Likewise, Behzad-Behbahani et al. reported higher anti-HHV-6 titers in MS patients' 


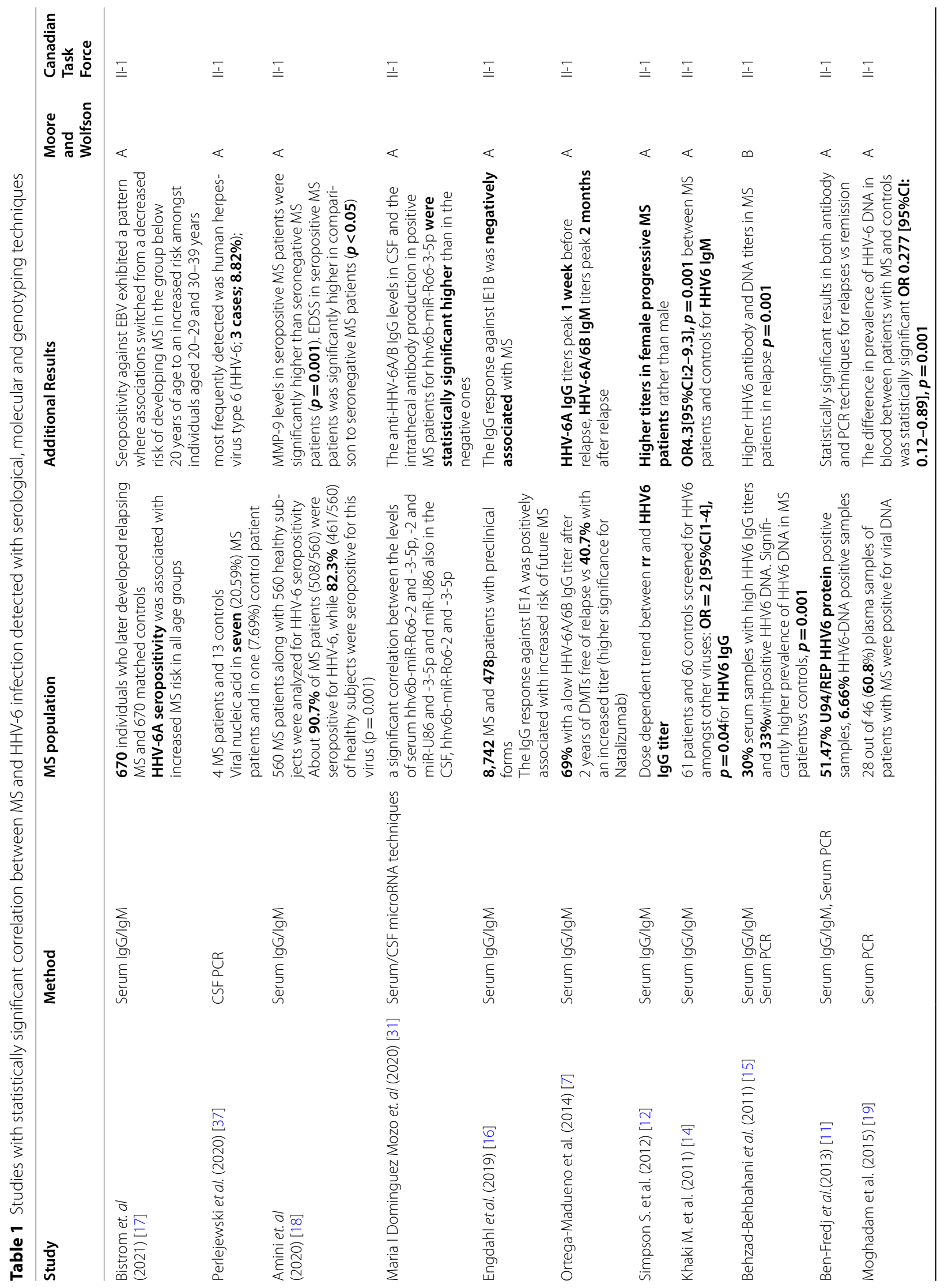




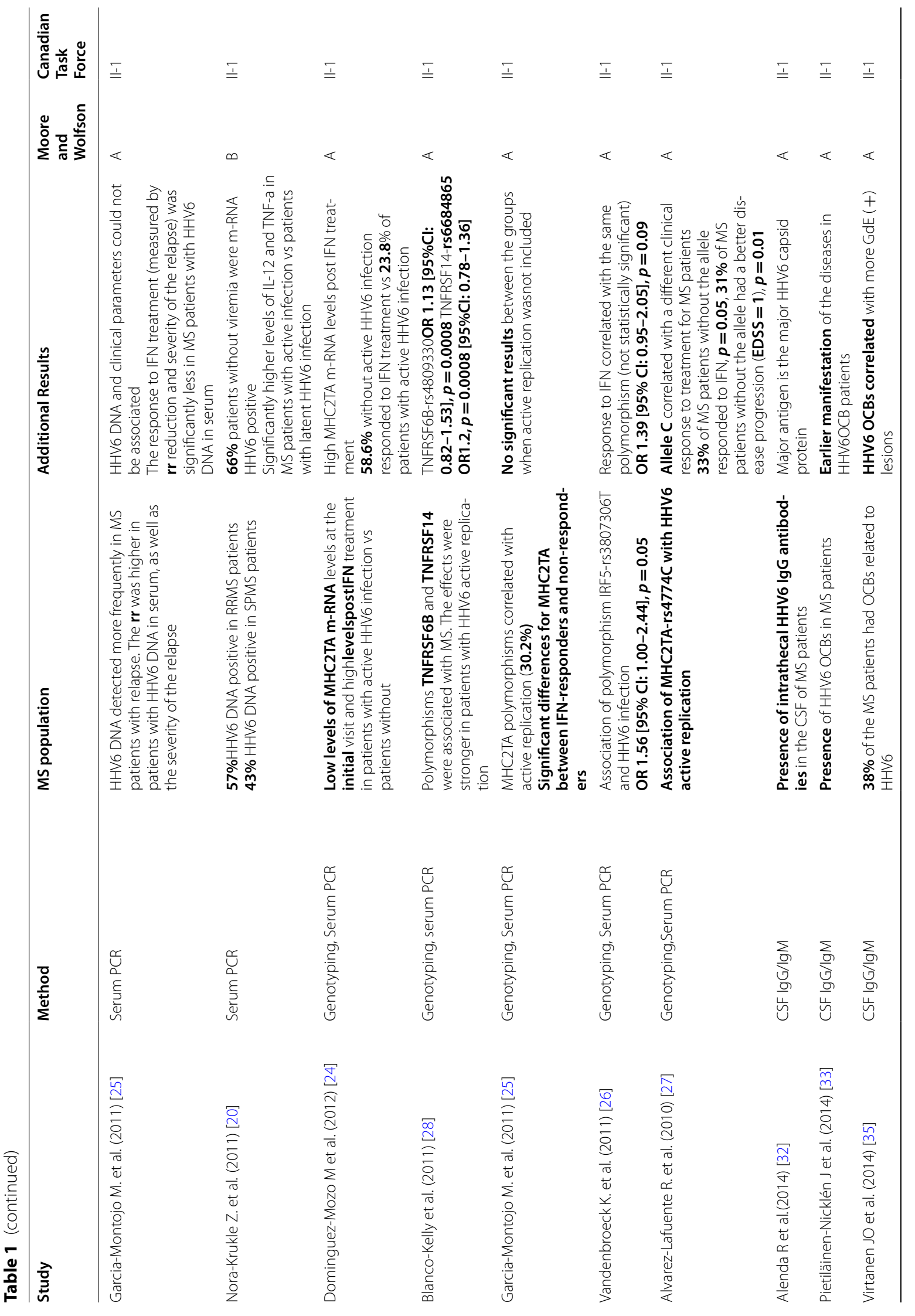




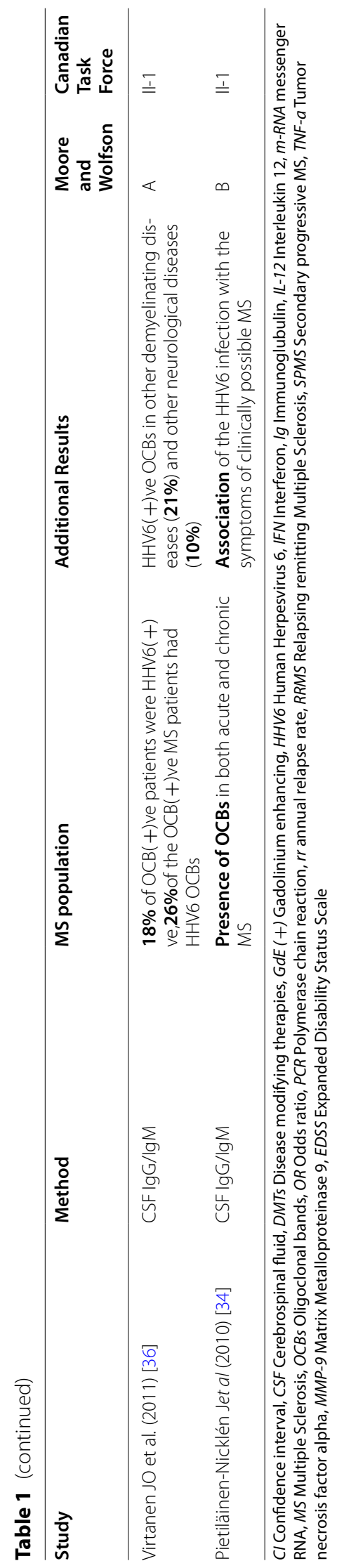




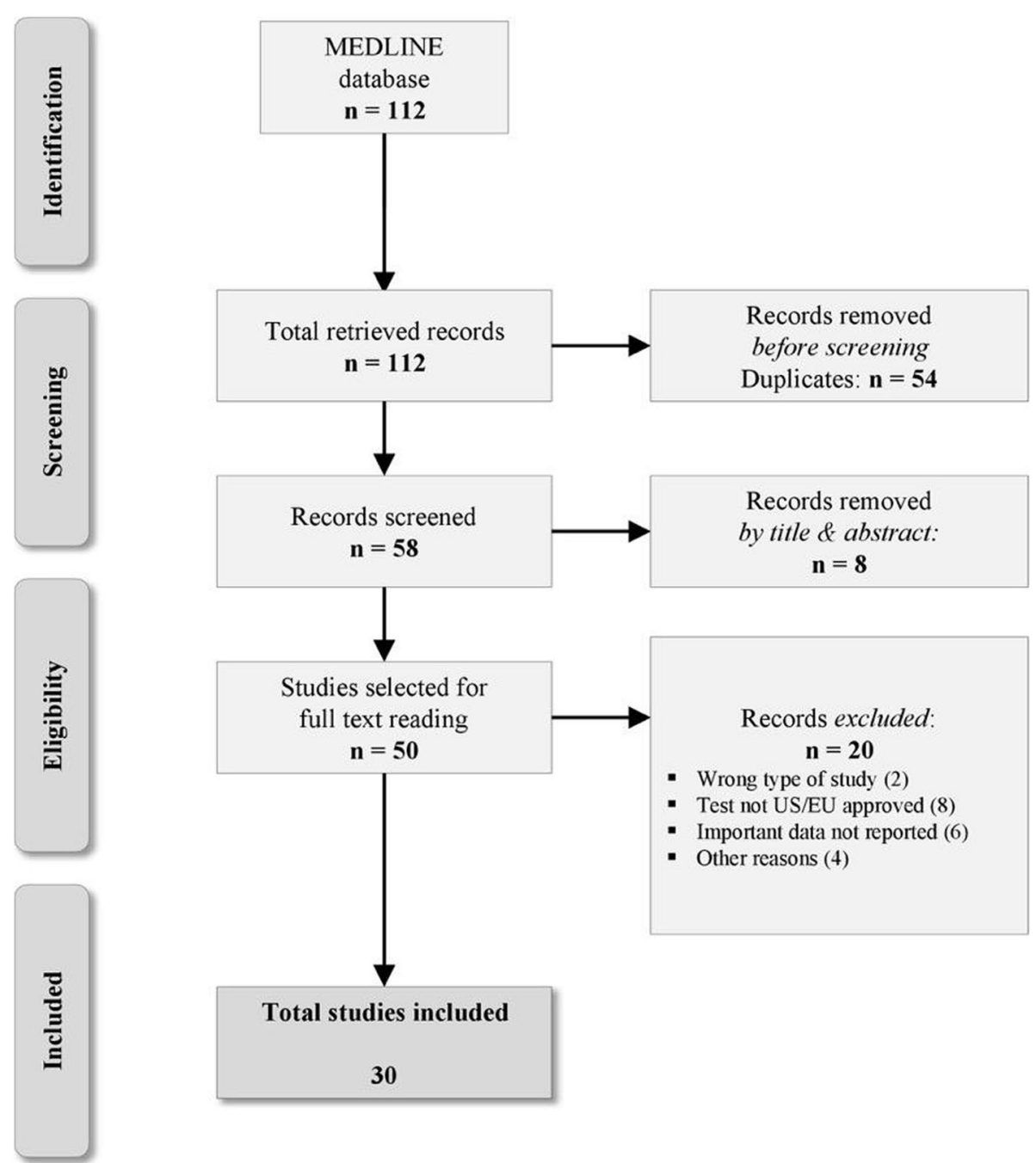

Fig. 2 Study selection Flow Chart

sera, especially during a clinical relapse [15]. Engdahl et al.found that the IgG response against the immediateearly 1 (IE1) protein of HHV-6 A was positively associated with MS [odds ratio (OR) $=1.55, p=9 \times 10^{-22}$ ], as well as with an increased risk for future development of MS $\left(\mathrm{OR}=2.22, p=2 \times 10^{-5}\right)$ [16]. In addition, Bistrom et al. found that HHV-6A was associated with increased MS risk in all age groups (total cohort odds ratio 2.1, 95\% confidence interval 1.6-2.7) [17] while Amini et al. demonstrated a significant HHV-6 seropositivity $(90.6 \%$ in $560 \mathrm{MS}$ patients) and significantly higher levels of Matrix Metalloproteinase 9 (MMP-9) in HHV-6 seropositive patients $(\mathrm{p}=0.001)$ as well as Expanded Disability Status Scale (EDSS) score [18].

As evidenced above, most studies show a significant difference in anti-HHV-6 antibody titers in MS patients compared to controls, significantly higher inflammatory markers higher EDSS disability scores, in more recent studies, and higher titers during clinical relapses. The main issue with the existing trials is the lack of a uniformly accepted positive titer threshold. Hence, positive results are interpreted based ondiverse criteria among studies. Moreover, seropositivity doesnot necessarily correlate with lymphoproliferation, as positive HHV- 6 antibodies are also detected in healthy controls. Finally, the interpretation of the positive IgM antibodies remains ambiguous, and may represent either recent infection or viral reactivation or even a latent or persistent subclinical infection which can be uncovered under certain circumstances, like immunosuppression.

\section{Polymerase Chain Reaction techniques}

The presence of HHV-6 genome in patients with MS was investigated in $\mathbf{1 4}$ studies using Polymerase Chain 
Reaction (PCR) techniques. Studies performing genotyping techniques are described separately. Among those, 12 found a positive correlation between the presence of viral nucleic acid and MS (Table 1). One study found that HHV-6 genomic sequences werepresentin 28 (60.8\%) out of 46 plasma samples taken from MS patients, compared to $13(28.2 \%)$ out of 46 positive samples in the control group $(\mathrm{OR}=0.277, p=0.0027)$ [19]. Similarly, another two studies performing both serologic and PCR techniques, reported higher prevalence of HHV-6 deoxyribonucleic acid (DNA) in MS patients compared to controls $[11,15]$. Additionally, a small study detected HHV-6 DNA in 4 out of 7 plasma samples of taken from patients with relapsing-remitting MS (RRMS) and in 4 out of 7 patients with secondary progressive MS (SPMS), during disease exacerbation, but not during remission; even though plasma viremia was undetectable in the remaining 3 RRMS patients, viral messenger ribonucleic acid (mRNA) transcription was detected in 2 of them [20]. In another study, the authors demonstrated that patients with detectable HHV-6 viremia had a higher risk of severe relapses and a worse response to Interferon beta (IFN-beta) therapy. Moreover, HHV-6 was detected more frequently during relapses, while patients with detectable HHV-6 in their sera had more frequent relapses, lower reduction of the relapse rate, and decreased proportion of responders to IFN-beta compared to those without an active viral replication [21]. Besides the studies reporting the prevalence of HHV-6 in blood and serum samples, the virus has been also isolated in urine samples fromMS patients. Indeed, Esmaili et al. reported a prevalence of HHV-6 DNA of $13.8 \%$ (23 out of 60) in urine samples obtained from patients with MS, whereas none of the 60 urine specimens from healthy controls was tested positive[22].

An important aspect which should be taken into account when investigating the detection of HHV-6 in blood or serum with PCR techniques is the phenomenon of chromosomal integration of human HHV-6 (ciHHV6). This is a condition whereby the complete HHV-6 genome is integrated into the host's germ line genome and is vertically transmitted in a Mendelian manner [23]. The condition is found in less than $1 \%$ of controls in the USA and the United Kingdom. HHV-6 levels in whole blood that exceed $5.5 \log 10$ copies/ml are strongly suggestive of ciHHV-6 [23]. Monitoring DNA load in plasma and serum is unreliable, both for identifying and for monitoring subjects with ciHHV-6 due to cell lysis and release of cellular DNA. High HHV-6 DNA loads associated with ciHHV-6 can lead to erroneous diagnosis of active infection [23].

Regarding studies performing genotyping techniques, Dominguez-Mozo et al. found that active replication of HHV-6 decreases the MHC2TA gene mRNA levels, a gene that plays a key role in controlling the immune response against viruses,and that low levels of serum HHV-6 DNA predict better response to IFN-beta treatment [24]. Likewise, Garcia-Montojo et al. demonstrated that MHC2TA polymorphisms correlated with active viral replication $(30.2 \%)$, while there were significant differences for MHC2TA between IFN-beta responders and non-responders in MS patients [25]. Two additional studies found a correlation between two frequently encountered gene polymorphisms in MS patients (IRF5rs3807306T and MHC2TA-rs 7447C), with active HHV-6 replication [26, 27]. Both, correlated HHV-6 active replication with low IFN-beta responsiveness. Another trial showed that TNFRSF6B-rs4809330(*)A and TNFRSF14rs6684865(*)A gene polymorphisms were associated with MS predisposition, and especially in patients with active HHV-6 replication [28].

Latham et al. found that the increased antiviral immune responses, directed primarily againstthe Epstein-Barr virus (EBV) virus, were significantly correlated with subsequent disease activity on Magnetic Resonance Imaging (MRI) scan in the form of Combined Unique Active lesions (CUAl). Regarding the HHV-6 the results were non-significant results [29]. Also, Ferrante et al. found a high frequency of HHV-6 DNA detection in peripheral blood mononuclear cells (PBMCs) isolated not only from patients with acute (41.6\%) and stable (22.2\%) MS, but also from healthy controls (45.9\%), without depicting a statistically significant difference among the three groups [30].

In the first study of its kind in the field of MS and HHV-6, Mozo and colleagues analyzed the microRNAs of HHV-6A: in paired samples of serum and CSF of 42 untreated MS patients and 23 patients with other neurological diseases using MicroRNA Assay techniques. Intrathecal $\mathrm{HHV}-6 \mathrm{~A} / \mathrm{B}$ antibody production and anti-HHV-6A/B IgG/IgM levels in serum were measured. In the serum of the whole population (MS and OND patients) they found a significant correlation between the levels of hhv6b-miR-Ro6-2 and -3-5p (Spearman $r=0.839$, pcorr $=3 \mathrm{E}-13$ ), -2 and miR-U86 (Spearman $r=0.578$, pcorr $=0.001$ ) and $-3-5 \mathrm{p}$ and miR-U86 (Spearman $r=0.698$, pcorr $=1.34 \mathrm{E}-5$ ); also in the CSF, between hhv6b-miR-Ro6-2 and -3-5p (Spearman $r=0.626$, pcorr $=8.52 \mathrm{E}-4)$. The anti-HHV-6A/B IgG levels in CSF and the intrathecal antibody production in positive MS patients for hhv6b-miR-Ro6-3-5p were statistical significantly higher than in the negative ones (pcorr $=0.006$ and $\operatorname{pcorr}=0.036)[31]$.

The above mentioned studies attempted to investigate the presence and the effects of HHV-6 active replication to the clinical course of MS as well as the responsiveness 
of the disease to IFN-beta treatment. All studies found an inverse correlation between HHV-6 replication and the patients' response to IFN-beta, which is probably suggesting that HHV-6 DNA may play a predictive role in treatment responsiveness and implying a possible association of viral active replication with the presence of IFN-beta neutralizing antibodies. The significance of the detectable viral DNA remains unclear, as reactivation does not always imply clinical infection. As such, these results should be interpreted cautiously.

\section{Cerebrospinal fluid studies}

The detection of anti-HHV-6 antibodies, oligoclonal bands (OCBs) and/or HHV-6 DNA in CSFwere investigated in 7 studies; all of them found a positive correlation between the presence of HHV-6 and MS and reported the presence of intrathecal HHV-6 OCBs in MS patients [32-36]. The frequency of positive CSF samples ranged between 18 and 38\%, while, the detection of HHV-6 DNA in the CSF was positively correlated with the amount of gadolinium enhanced $(\mathrm{GdE}+)$ lesions in the MRI [32-36]. In a more recent HHV-6 DNA detection study Perlejewski et al. searched for viral RNA and DNA in the CSFof $34 \mathrm{MS}$ patients and 13 controls using RT$\mathrm{PCR} / \mathrm{PCR}$. It revealed the presence of viral nucleic acid in seven (20.59\%) MS patients and in one (7.69\%) control patient. In MS patients the most frequently detected was human herpesvirus type 6 (HHV-6; 3 cases; $8.82 \%$ ); followed by Epstein-Barr virus (EBV; 2 cases; $5.88 \%$ ), varicella zoster virus (VZV; 1 case; $2.94 \%)$ and Enterovirus (EV; 1 case; $2.94 \%$ ) [37].

Both IgG and intrathecal production and OCBs can be detected in the CSF of MS patients. However, the major difference between themis that intrathecal IgG production is polyclonal while OCBs are a marker of clonal antigen-specific activation. Thus, OCBs allow the identification of a disease relevant antigen [32]. Consequently, it has been attempted to correlate HHV-6 with the formation of OCBs in MS.An equally interesting theory is that HHV-6 OCBs remain the same over the course of MS. Previous data suggested that MS patients with HHV-6 or EBV OCBs had fewer GdE + lesions [38]. One study demonstrated that HHV-6 DNA in CSF was associated with the number of $\mathrm{GdE}+$ lesions, invigorating an interesting hypothesis that HHV-6 OCBs in CSF control the viral activity within the CNS and limit the damage to brain tissue due to viral activation within the CNS [35]. This hypothesis is consistent with the findings that both HHV-6 antibody titers and DNA levels are higher in serum during a clinical relapse [15]. However, one must bear in mind that in many cases CSF studies are performed based on the availability of the CSF samples. Consequently, it is quite burdensome, or even impossible, to achieve a careful cross match between MS and control samples.

\section{Discussion}

The vast majority (27 out of 30 ) of the studies presented in this review concluded on a significant association between HHV-6 detection and various clinical aspects of MS, implying a possible pathogenetic linkage between them. However, there is a different interpretation of the same findings as presented above.

A breakdown in the Blood Brain Barrier (BBB) during an acute infection with $\mathrm{HHV}-6$, may result in the same antibody detection in sera or CSF samples. In both cases the antibodies will originate from the periphery which, of course, does not suggest a direct implication of HHV-6 in the pathogenesis of MS. In addition, there are many alternative scenarios that theoretically may lead to the presence of HHV-6 antibodies in the biological fluids of MS patients. Some of those are reactivation of a latent HHV-6 infection, a subclinical infectious course without any clinical symptoms to i.e. immunocompromised patients and immune system hyperactivity in the case of MS relapse. Moreover, a positive sample for HHV-6 antibodies can be the result of an infection in childhood or an active infection in adult life. It remains to be seen whether these two conditions can have the same influence in pathogenesis of MS, given the fact that their chronologic encounter is quite different. Conclusively, HHV-6 antibodies studies highlight the importance of a positive sample no matter what the mechanism behind this positivity is.

PCR techniques can give an answer to the previous questions given the fact that they can identify actual viral replication. However, PCR HHV-6 specific techniques cannot distinguish between an active new infection from the reactivation of a latent disease or a chromosomal integration which requires special tissue testing. It remains to be answered whether these three conditions may cause the same immune hyperactivity and more pronounced neurological symptoms. The quantification of the exact viral load may shed some light to this problem as active infection is associated with higher viral load. In addition, it may contribute to the clarification of whether reactivation of a latent infection or chromosomal integration may have the same impact as an active new infection to the pathogenesis of MS.

Moreover, there are many alternative scenarios that theoretically may lead to the presence of HHV-6 antibodies in the biological fluids of MS patients. Some of those are reactivation of a latent $\mathrm{HHV}-6$ infection, a subclinical infectious course without any clinical symptoms but to i.e. immunocompromised patients and immune system hyperactivity in the case of MS relapse. 
In addition, a positive sample for HHV-6 antibodies can be the result of an infection in childhood or an active infection in adult life. It remains to be seen whether these two conditions can have the same influence in pathogenesis of MS given the fact that their chronologic encounter is very different.

Ultimately and in order to tackle with the above conditions and alternative interpretations of the same results, a controlled clinical trial of an efficacious and CNS penetrable anti HHV-6 drug may be the only way to ascertain the actual role of HHV- 6 to MS. The authors think that robust clinical results in favor of a positive impact on the clinical, radiological and less likely disability parameters of MS would contribute to the adaptation of a significant environmental MS pathogenetic role while ambiguous or negative results will add to the existing controversy.

In any case, the authors think that there must be certain conditions fulfilled so as to facilitate a solid trial investigating the pathogenetic role of HHV-6.

A. The controls used should be as similar to MS patients: Whenever possible the researchers have to ensure that the only difference between the research group and the control group is the well-established diagnosis of MS. Apart from the apparent age, sex and race cross match it is imperative for the control participants to come from the same population pool as the MS group participants. This is to ensure that the genetic background especially regarding the various polymorphisms related with MS that in many cases are population specific will be the same for the two groups. As a result, the interactions between environmental factors (HHV-6) and the genome (samples from the same population and consequently same prevalence of polymorphisms related to MS) wont influence the possible HHV-6 role to MS.

B. Participants in the MS group have to have welldefined diagnostic criteria and ideally to be matched according to disease onset. A research group consisted of MS patients with robust diagnostic criteria and as much as possible similar disease onset, which means similar disease profile and clinical course, will ensure investigating the possible role of HHV-6 on the same substrate, where similar pathogenetic mechanisms take place. Inclusion of patients with progressive forms of the disease for example, does not necessary implicate the presence of the virus, in the case of a positive result, during the time of diagnosis or that its presence was more than a chance event.

C. Universal HHV-6 sample positivity threshold has to be established so as the research criteria to be the same in all studies. It is also essential to present all negative samples as well to rule out the possibility of a group of negative controls due to an experimental role. Finally, it is preferred that the results to be evaluated by observers blinded to the diagnosis.

Two important aspects regarding the HHV-6 is the HHV-6 biology and the HHV-6 interactions with the genome.

Another aspect that needs to be noted is based on the study of Leibovitch et al. which proved that HHV-6A DNA is more prevalent than HHV-6B in saliva samples of MS patients vs controls. This is probably because HHV$6 \mathrm{~A}$ is more neurotropic than HHV-6B [39]. As a consequence, the authors think that it is important to fully investigate the properties of every virus and elucidate their effect on the CNS. Hence, all future studies have to distinguish between the various types of HHV-6 as they seem to exhibit different behavior. This may cause different antibody reactivity, behavior and even time of acquisition of HHV-6. Different types of HHV-6 may mean different time threshold of a positive sample.

Interaction of the HHV-6 with the genome is also a very promising field in the HHV-6 pathogenetic research. In every study there is a correlation with MS related polymorphisms or genes. Is this perhaps due to specific areas in the HHV-6 genome in MS patients? And if that is the case are there different genome areas in HHV-6 DNA in MS patients and controls? Or is this location specific? Different HHV-6 genome sequence in CNS located HHV-6 and in HHV-6 located in the periphery? After all we are discussing about a virus with ubiquitous nature which is hypothesized to be implicated in a nonubiquitous inflammatory pathogenetic mechanism. Perhaps the reason behind this paradox is genetic variability of HHV-6.

The evidence regarding the pathogenetic role of HHV-6 is solid and engulfs several different methodological approaches. Future research needs to be designed to answer all the methodological and pathophysiological queries that have aroused based on the existing data. HHV-6 antiviral medication trials will also contribute immensely in the clarification of the nature of HHV-6's role in the pathogenesis of MS.

\section{Abbreviations \\ BBB: Blood Brain Barrier; CiHHV-6: Chromosomal integration of HHV-6; CNS: Central Nervous System; CSF: Cerebrospinal Fluid; DMT: Disease Modifying Therapy; DNA: Deoxyribonucleic acid; EBV: Epstein-Barr Virus; EDSS: Expanded Disability Status Scale; GdE+: Gadolinium Enhanced Lesion; HHV-6: Human Herpes Virus 6; IFN-Beta: Interferon Beta; MMP-9: Matrix Metalloproteinase 9; MRI: Magnetic Resonance Imaging; mRNA: RNA messenger; MS: Multiple Scle- rosis; OCB: Oligoclonal Bands; OR: Odds Ratio; PCR: Polymerase Chain Reaction; RNA: Ribonucleic acid.}

Acknowledgements

Nothing to acknowledge 


\section{Authors' contributions}

D.Kitsos, P.C.Fragkou and K. Foska performed the literature search; K.I Voumvourakis and S.Tsiodras completed the quality classification of the studies and their methodological limitations. All authors read and approved the final manuscript.

\section{Funding}

Nil funding received

\section{Availability of data and materials}

The datasets used and/or analyzed during the current study available from the corresponding author on reasonable request.

\section{Declarations}

\section{Ethics approval and consent to participate}

Not applicable

\section{Consent for publications}

Not applicable

\section{Competing interests}

Not applicable

\section{Author details}

'2nd Department of Neurology, Attikon University Hospital, National and Kapodistrian University of Athens, Athens, Greece. ${ }^{2} 4$ th Department of Internal Medicine, Attikon University Hospital, National and Kapodistrian University of Athens, Athens, Greece.

Received: 23 February 2021 Accepted: 22 October 2021

Published online: 15 February 2022

\section{References}

1. Compston A, Coles A. Multiple sclerosis. Lancet. 2008;372(9648):1502-17.

2. Panitch HS. Influence of infection on exacerbations of multiple sclerosis. Ann Neurol. 1994;36(suppl):S25-8

3. Sibley WA, Bamford CR, Clark K. Clinical viral infections and multiple sclerosis. Lancet. 1985;1(8441):1313-5.

4. Voumvourakis KI, Kitsos DK, Tsiodras S, et al. Human herpesvirus 6 infection as a trigger of multiple sclerosis. Mayo Clin Proc. 2010;85(11):102330. https://doi.org/10.4065/mcp.2010.0350.

5. Moore FG, Wolfson C. Human herpes virus 6 and multiple sclerosis. Acta Neurol Scand. 2002;106(2):63-83.

6. Canadian Task Force on the Periodic Health Examination. The periodic health examination. Can Med Assoc J. 1979;121(9):1193-254.

7. Ortega-Madueño I, Garcia-Montojo M, Dominguez-Mozo MI, et al. Anti-human herpesvirus 6A/B lgG correlates with relapses and progression in multiple sclerosis. PLoS One. 2014;9(8):e104836 Published 2014 Aug 11

8. Stüve O, Marra CM, Jerome KR, Selter RC, Biberacher V, Grummel V, et al. Immune surveillance in multiple sclerosis patients treated with natalizumab. Ann Neurol. 2006:59:743-7.

9. Selter RC, Biberacher V, Grummel V, et al. Natalizumab treatment decreases serum lgM and lgG levels in multiple sclerosis patients. MultScler. 2013;19:1454-61.

10. Warnke C, Stettner M, Lehmensiek V, et al. Natalizumab exerts a suppressive effect on surrogates of B cell function in blood and CSF. MultScler. 2015;21:1036-44

11. Ben-Fredj N, Ben-Selma W, Rotola A, et al. Prevalence of human herpesvirus U94/REP antibodies and DNA in Tunisian multiple sclerosis patients. J Neurovirol. 2013:19(1):42-7.

12. Simpson S Jr, Taylor B, Dwyer DE, et al. Anti-HHV-6 IgG titer significantly predicts subsequent relapse risk in multiple sclerosis. MultScler. 2012;18(6):799-806.

13. Simpson S, Jr,TaylorB.Burrows J, et al. EBV \& HHV6 reactivation is infrequent and not associated with MS clinical course. Acta Neurologica Scandinavica. 2014;130(5):328-37. https://doi.org/10.1111/ane.12268.
14. Khaki M, Ghazavi A Ghasami K et al. Evaluation of viral antibodies in Iranian multiple sclerosis patients. Neurosciences (Riyadh). 2011;16(3):224-8.

15. Behzad-Behbahani A, Mikaeili MH, Entezam M, et al. Human herpesvirus-6 viral load and antibody titer in serum samples of patients with multiple sclerosis. J Microbiol Immunol Infect. 2011:44(4):247-51.

16. Engdahl E, Gustafsson R, Huang J, et al. Increased Serological Response Against Human Herpesvirus 6A Is Associated With Risk for Multiple Sclerosis. Front Immunol. 2019;10:2715. https://doi.org/10.3389/fimmu.2019. 02715 Published 2019 Nov 26.

17. Biström $M$, Jons D, Engdahl E, et al. Epstein-Barr virus infection after adolescence and human herpesvirus $6 \mathrm{~A}$ as risk factors for multiple sclerosis. Eur J Neurol. 2021;28(2):579-86.

18. Amini R, Karampoor S, Zahednasab H, Keyvani H, et al. Serum levels of matrix metalloproteinase-2, -9 , and vitamin $D$ in patients with multiple sclerosis with or without herpesvirus-6 seropositivity. Braz J Infect Dis. 2020;24(2):144-9.

19. Ahmadi JalaliMoghadam M, Honarmand H. Human Herpesvirus 6 Viremia in Patients with Classic Multiple Scelerosis in Guilan, Iran. JoMMID. 2014;2(4):133-7.

20. Nora-KrukleZ., Chapenko S., Logina I. Millers, et al. Human Herpesvirus 6 and 7 Reactivation and Disease Activity in Multiple Sclerosis. Medicina (Kaunas). 2011;47:527-31. https://doi.org/10.3390/medicina47100075.

21. Garcia-Montojo M, De Las HV, Dominguez-Mozo M, et al. Human herpesvirus 6 and effectiveness of interferon $\beta 1 \mathrm{~b}$ in multiple sclerosis patients. Eur J Neurol. 2011:18(8):1027-35.

22. Esmaili K, Amini K. Detection of Human Herpes Virus Type 6 and Varicella Zoster Virus in the Urine of Patients with Multiple Sclerosis in Kerman Province. Iran Shefaye Khatam. 2018;6(3):19-24.

23. Pellett PE, Ablashi DV, Ambros PF, et al. Chromosomally integrated human herpesvirus 6: questions and answers. Rev Med Virol. 2012;22(3):144-55. https://doi.org/10.1002/rmv.715.

24. Dominguez-Mozo MI, Garcia-Montojo M, De Las Heras V, et al. MHC2TA mRNA levels and human herpesvirus 6 in multiple sclerosis patients treated with interferon beta along two-year follow-up. BMC Neurol. 2012;12:107 Published 2012 Sep 25

25. Garcia-Montojo M, Martinez A, De Las HV, et al. Herpesvirus active replication in multiple sclerosis: a genetic control? J Neurol Sci. 2011:311(1-2):98-102.

26. Vandenbroeck K, Alloza I, Swaminathan B, et al. Validation of IRF5 as multiple sclerosis risk gene: putative role in interferon beta therapy and human herpes virus-6 infection. Genes Immun. 2011;12:40-5.

27. Alvarez-Lafuente R, Martinez A, Garcia-Montojo M, et al. MHC2TA rs4774C and HHV-6A active replication in multiple sclerosis patients. Eur J Neurol. 2010;17:129-35.

28. Blanco-Kelly F, Alvarez-Lafuente R, Alcina A, et al. Members 6B and 14 of the TNF receptor superfamily in multiple sclerosis predisposition. Genes Immun. 2011;12(2):145-8. https://doi.org/10.1038/gene.2010.42.

29. Latham L.B., Lee M.J., LincolnJ A, et al. Antivirus immune activity in multiple sclerosis correlates with MRI activity. Acta NeurolScand. 2016;133:17-24.

30. Ferrante $P$, Mancuso R, Pagani E, et al. Molecular evidences for a role of HSV-1 in multiple sclerosis clinical acute attack. J Neurovirol. 2000;6(Suppl 2):S109-14.

31. Domínguez-Mozo MI, Nieto-Guerrero A, Pérez-Pérez S, García-Martínez MÁ, Arroyo R, Álvarez-Lafuente R. MicroRNAs of Human Herpesvirus 6A and $6 \mathrm{~B}$ in Serum and Cerebrospinal Fluid of Multiple Sclerosis Patients. Front Immunol. 2020;18(11):2142.

32. Alenda R, Álvarez-Lafuente R, Costa-Frossard L, et al. Identification of the major HHV-6 antigen recognized by cerebrospinal fluid lgG in multiple sclerosis. Eur J Neurol. 2014:21(8):1096-101.

33. Pietiläinen-Nicklén J, Virtanen JO, Uotila L, et al. HHV-6-positivity in diseases with demyelination. J Clin Virol. 2014:61(2):216-9.

34. Pietiläinen J, Virtanen JO, Uotila L, et al. HHV-6 infection in multiple sclerosis. A clinical and laboratory analysis. Eur J Neurol. 2010;17:506-9.

35. Virtanen JO, Wohler J, Fenton K, et al. Oligoclonal bands in multiple sclerosis reactive against two herpesviruses and association with magnetic resonance imaging findings. MultScler. 2014;20(1):27-34

36. Virtanen JO, Pietiläinen-Nicklén J, Uotila L, et al. Intrathecal human herpesvirus 6 antibodies in multiple sclerosis and other demyelinating diseases presenting as oligoclonal bands in cerebrospinal fluid. J Neuroimmunol. 2011:237(1-2):93-7. 
37. Perlejewski K, Bukowska-Ośko I, Rydzanicz M, et al. Search for viral agents in cerebrospinal fluid in patients with multiple sclerosis using real-time PCR and metagenomics. PLoS One. 2020;15(10):e0240601.

38. Panitch HS. Influence of infection on exacerbations of multiple sclerosis. Ann Neurol. 1994;36(suppl):S25-8.

39. Leibovitch EC, Jacobson S. Evidence linking HHV-6 with multiple sclerosis: an update. CurrOpinVirol. 2014;9:127-33.

\section{Publisher's Note}

Springer Nature remains neutral with regard to jurisdictional claims in published maps and institutional affiliations.

- fast, convenient online submission

- thorough peer review by experienced researchers in your field

- rapid publication on acceptance

- support for research data, including large and complex data types

- gold Open Access which fosters wider collaboration and increased citations

- maximum visibility for your research: over 100M website views per year

At BMC, research is always in progress.

Learn more biomedcentral.com/submissions 